\title{
On the Synchronization of Oscillators Coupled by One Negative Resistor
}

\author{
Hiroyuki Kanasugi $\dagger$, Seiichirou Moro $\dagger$, Yoshifumi Nishio $\ddagger$ and Shinsaku Mori $\dagger$ \\ † Department of Electrical Engineering, \\ Keio University \\ 3-14-1 Hiyoshi, Kohoku, \\ Yokohama, 223 JAPAN \\ Tel: (+81) 45-563-1141 Ext. 3319 \\ Email: kana@mori.elec.keio.ac.jp \\ $\ddagger$ Department of Electrical and Electronic \\ Engineering, Tokushima University \\ 2-1 Minami-Jousanjima, \\ Tokushima, 770 JAPAN \\ Email: nishio@ee.tokushima-u.ac.jp
}

\begin{abstract}
There have been many investigations of the mutual synchronization of oscillators. In this study we consider $N$ oscillators which have the same natural frequency, mutually coupled by one negative resistor. In this system, according to the negative range of the coupling negative resistor, we can observe various interesting synchronization phenomena. Because this system tends to minimize the power consumption in the coupling negative resistor. Especially when $i-v$ characteristics of the coupling negative resistor are not third-power, but linear and negative, this system tends to be stable by producing the power in the coupling one. The phase states are changed accordeing to the negative range of the coupling negative resistor.
\end{abstract}

\section{INTRODUCTION}

There have been many investigations of the mutual synchronization of oscillators([1]-[7] and therein). Kimura and Mano have confirmed that two oscillators coupled by one positive resistor are synchronized at opposite phase, and that the system tends to minimize the power consumption in the coupling positive resistor[1]. Endo et al have analyzed a large number of coupled van der Pol oscillators [2]-[4], and we have confirmed that $N$-phase oscillation $(N=2 \sim 13)$ can be stably excited for the case that the nonlinearity of each oscillator is strong [5][6]. In such systems various interesting synchronization phenomena are observed. Because the system tends to minimize the power consumption in the coupling positive resistor, i.e., the system tends to minimize the current through the coupling one, and the sum of output voltage $\left(x_{1} \sim x_{N}\right)$. For the case with third-power nonlinear characteristics, all oscillators exhibit the oscillation. And $N$-phase oscillation can be stably excited, because of taking any phase differences among $\phi=2 k \pi / N(k=1,2, \cdots, N-1)$. For the case that $N$ is not a prime number, $N$-phase oscillation seems to be difficult to observe and another types of synchronization are observed.

In this study, we investigate synchronization phenom-

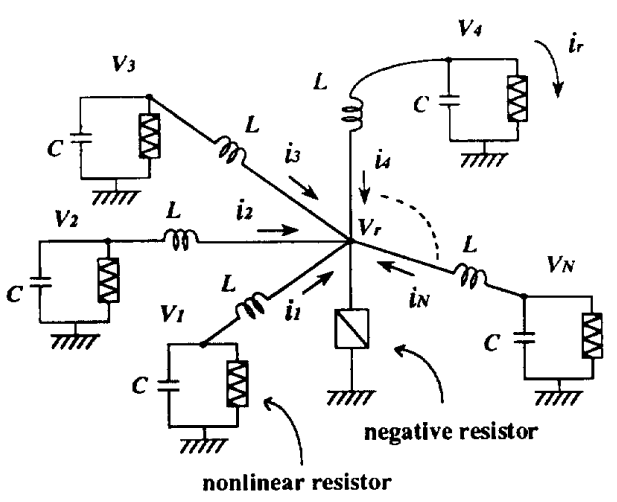

(a)

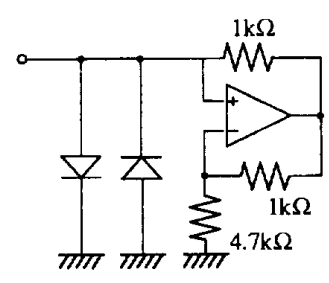

(b)

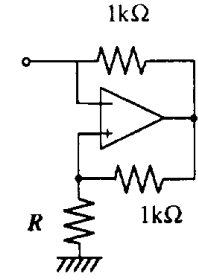

(c)
Figure 1: Circuit Model. (a) Oscillators coupled by one negative resistor. (b) Realization of nonlinear resistor. (c) Realization of the coupling negative resistor.

ena observed from $N$ oscillators with the same natural frequency, mutually coupled by one negative resistor. This system tends to minimize the power consumption in the coupling negative resistor, just as the positive resistor. Then various interesting synchronization phenomena is observed. When $i-v$ characteristics of the coupling negative resistor are not third-power, but linear and negative, this system tends to be stable by producing the power in the coupling one. i.e., the system tends to be maximum the current through the coupling one, and the sum of output voltage $\left(x_{1} \sim x_{N}\right)$. As a result, when two, 
three or more oscillators are coupled, each oscillator of this system are synchronized at the in-phase, each other. On the other hand, when $i-v$ characteristics of the coupling negative resistor are third-power, this system tends to be stable by producing the power in the negative range of the coupling one, and by consuming the power in the positive range. As a result, according to the negative range of the coupling one, the phase states is changed. Especially when the negative range is very small, we can observe $N$-phase oscillation.

We hope that our results can be applied to the design of some kinds of phase control systems in near future.

\section{CIRCUIT MODEL}

The circuit model is shown in Figure 1. The circuit equation is described as follows,

$$
\begin{aligned}
C \frac{d v_{k}}{d t} & =-i_{k}-i_{r}\left(v_{k}\right) \\
L \frac{d i_{k}}{d t} & =v_{k}-v_{r} \\
(k & =1,2, \cdots, N)
\end{aligned}
$$

where $i_{r}\left(v_{k}\right)$ means $v-i$ characteristics of nonlinear resistor, and $v_{r}$ means $i-v$ characteristics of the coupling negative resistor. In our system, these are approximated by the following function respectively.

$$
\begin{aligned}
i_{r}\left(v_{k}\right) & =-g_{1} v_{k}+g_{3} v_{k}^{3} \quad\left(g_{1}, g_{3}>0\right) \\
v_{r} & =-r_{1} \sum_{j=1}^{N} i_{j}+r_{3}\left(\sum_{j=1}^{N} i_{j}\right)^{3}\left(r_{1}, r_{3}>0\right)
\end{aligned}
$$

By changing the variables,

$$
\begin{array}{r}
t=\sqrt{L C} \tau, \\
v_{k}=\sqrt{\frac{g_{1}}{3 g_{3}}} x_{k}, \quad i_{k}=\sqrt{\frac{g_{1}}{3 g_{3}}} \sqrt{\frac{C}{L}} y_{k}, \\
\alpha=r_{1} \sqrt{\frac{C}{L}}, \quad \beta=g_{1} \sqrt{\frac{L}{C}}, \quad \gamma=\frac{g_{1} r_{3}}{g_{3}}\left(\sqrt{\frac{C}{L}}\right)^{3}
\end{array}
$$

(1) is normalized as

$$
\begin{gathered}
\dot{x}_{k}=-y_{k}+\beta\left(x_{k}-\frac{1}{3} x_{k}^{3}\right) \\
\dot{y}_{k}=x_{k}+\alpha \sum_{j=1}^{N} y_{j}-\frac{\gamma}{3}\left(\sum_{j=1}^{N} y_{j}\right)^{3} \\
(k=1,2, \cdots, N)
\end{gathered}
$$

where $\alpha$ is the coupling factor and $\beta$ is the largeness of nonlinearity. When $\beta$ is small, the oscillation of each oscillator can be regarded as almost purely sinusoidal. And $\gamma$ is the negative range of the coupling negative resistor. When $\gamma$ is small, the negative range is large. Especially when $\gamma=0, i-v$ characteristics of the coupling negative resistor are not third-power, but linear and negative.

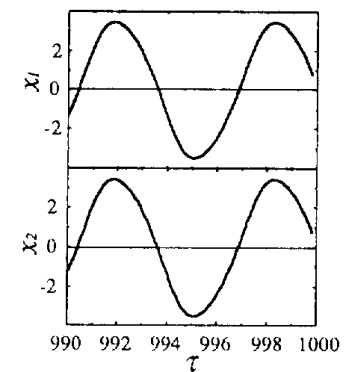

(a) $N=2$

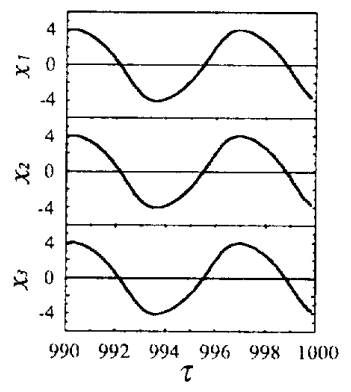

(a) $N=3$

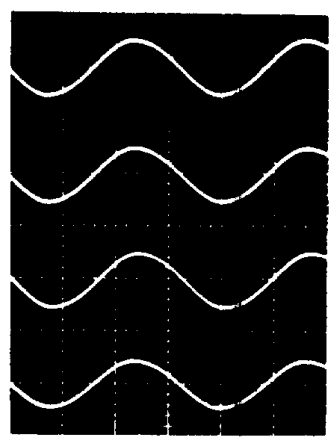

(b) $N=4$

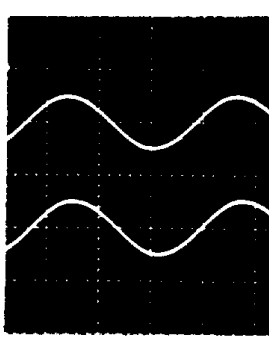

(b) $N=2$

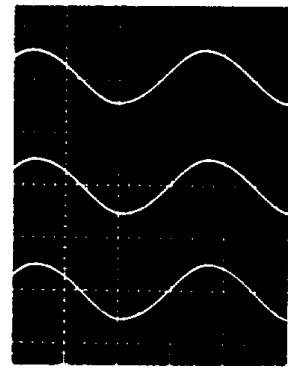

(b) $N=3$

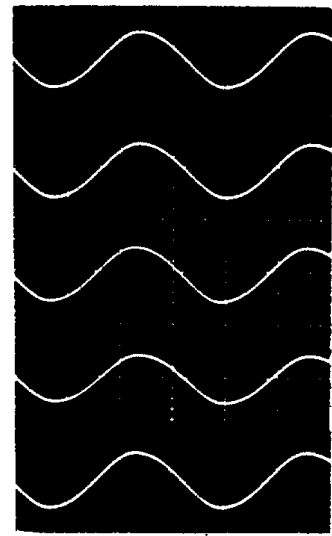

(b) $N=5$
Figure 2: (a) Numerical results for the case that $N=2,3$. $\alpha=0.1, \beta=0.1, \gamma=0, \Delta \omega_{1}=0, \Delta \omega_{2}=0.001, \Delta \omega_{3}=0.002$ ) (b) Experimental results for the case that $N=2 \sim 5$. ( $L=10 \mathrm{mH}$, $C=0.068 \mu \mathrm{F}, R=4.7 \Omega$, horizontal scale: $50 \mu \mathrm{sec} /$ div., vertical scale: $1.0 \mathrm{~V} /$ div. )

\section{THEORETICAL ANALYSIS}

The synchronization phenomena in this system can be analyzed theoretically by using averaging method. In this system, because nonlinear characteristics of all oscillators is approximated by third-power, all oscillators exhibit the oscillation. At first, we consider the case that $\gamma=0, N=$ 2. When $\beta$ is small, we can get the following equations 
about the amplitude $a_{k}$ and the phase $\theta_{k}$.

$$
\begin{gathered}
\dot{a}_{k}=\frac{a_{k}}{2}\left(1-\frac{1}{4} a_{k}^{2}\right) a_{k}+\frac{\alpha}{2} \sum_{j=1}^{N} a_{j} \cos \left(\theta_{k}-\theta_{j}\right) \\
\dot{\theta_{k}=}-\frac{\alpha}{2 a_{k}} \sum_{j=1}^{N} a_{j} \sin \left(\theta_{k}-\theta_{j}\right) \\
(k=1,2, \cdots, N)
\end{gathered}
$$

In this study, the amplitudes has less effect for stability of steady states, so we only consider the equations on the phases. Then we can get the steady states of the phase difference $\theta_{0}$.

$$
\theta_{0}=2 n \pi \quad(n=0, \pm 1, \pm 2, \cdots)
$$

So we can see that $x_{1}$ and $x_{2}$ is synchronized at the inphase, each other for $\gamma=0, N=2$. And we consider the case that $\gamma=0, N=3$ and $\gamma$ is very small, $N=2$. Then it is stable when the phase difference of each oscillator is 0 , just as $\gamma=0, N=2$. So we can take in-phase oscillations respectively.

\section{NUMERICAL CALCULATIONS AND CIRCUIT EXPERIMENTS}

In this section, we confirm the synchronization phenomena by numerical calculations and circuit experiments. For numerical calculations by using the RungeKutta-Gill method, in order to consider the difference between the natural frequencies of real oscillators, (5) is rewritten as follows,

$$
\begin{aligned}
\dot{x}_{k}= & -y_{k}+\beta\left(x_{k}-\frac{1}{3} x_{k}^{3}\right) \\
\dot{y}_{k}= & \left(1+\Delta \omega_{k}\right) x_{k}+\alpha \sum_{j=1}^{N} y_{j}-\frac{\gamma}{3}\left(\sum_{j=1}^{N} y_{j}\right)^{3} \\
& \Delta \omega_{k}=(k-1) \times 10^{-3} \\
& (k=1,2, \cdots, N)
\end{aligned}
$$

On the other hand for circuit experiments, in order to use the coupling negative resistor in linear and negative range, we connect two diodes in parallel as shown in Figure 1(b). Then each of oscillators exhibit the oscillation with the small amplitude, and the frequency of each oscillator is almost $6.093 \mathrm{kHz}$. The circuit as shown in Figure $1(\mathrm{c})$ is realized the coupling negative resistor. If we change higher resistor than $1 \mathrm{k} \Omega$ resistor, the negative range of the coupling one is smaller.

Figure 2 shows the numerical results and the corresponding results of circuit experiments in the case that $\gamma=0$, i.e.,$i-v$ characteristics of the coupling negative resistor are not third-power, but linear and negative. From these results, we confirmed that in-phase steady states are stably excited for all numbers of $N$.

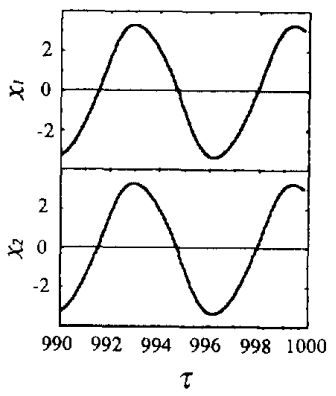

(a) $N=2$

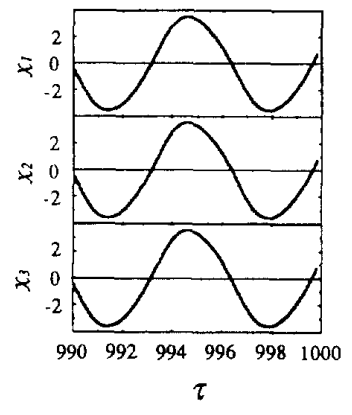

(a) $N=3$

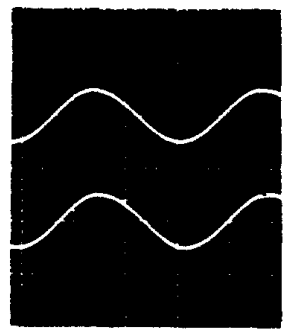

(b) $N=2$

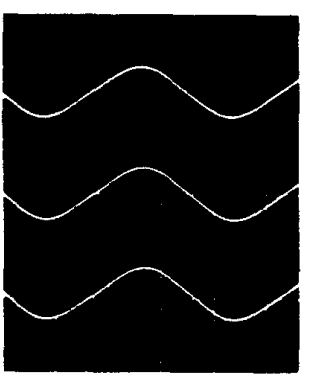

(b) $N=3$
Figure 3: (a) Numerical results for the case that $N=2,3$. ( $\alpha=0.1, \beta=0.1, \underline{\gamma=0.001}, \Delta \omega_{1}=0, \Delta \omega_{2}=0.001, \Delta \omega_{3}=0.002$ ) (b) Experimental results for the case that $N=2,3$. ( $L=10 \mathrm{mH}$, $C=0.068 \mu \mathrm{F}, R=42 \Omega$, horizontal scale: $50 \mu \mathrm{sec} /$ div., vertical scale: $1.0 \mathrm{~V} /$ div. )

Figure $3 \sim 5$ shows the case that $i-v$ characteristics of the coupling negative resistor are third-power. In Figure 3 , because the negative range of the coupling negative resistor is much larger than the positive range, two or three signals seem to be almost synchronized at the in-phase. In Figure 4, because this system tends to be stable by producing the power in the negative range of the coupling one, and by consuming the power in the positive range, two or three signals are synchronized at the different phase. In Figure 5, because the negative range of the coupling negative resistor is much smaller than the positive range, two or three signals seem to be almost synchronized at the $N$-phase. That is just like synchronization phenomena when $N$ oscillators coupled by one positive resistor.

From both of numerical calculations and circuit experiments we confirmed various interesting synchronization phenomena according to the negative range of the coupling negative resistor.

\section{CONCLUSION}

In this study we have investigated $N$ oscillators which have the same natural frequency, mutually coupled by 

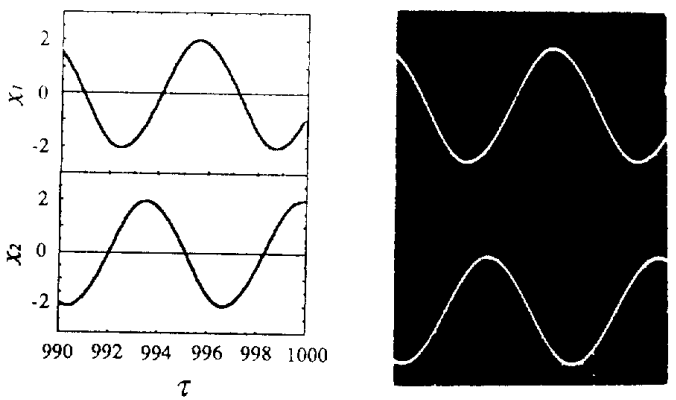

(a) $N=2$

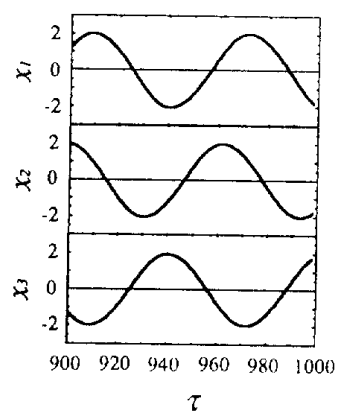

(a) $N=3$ (b) $N=2$

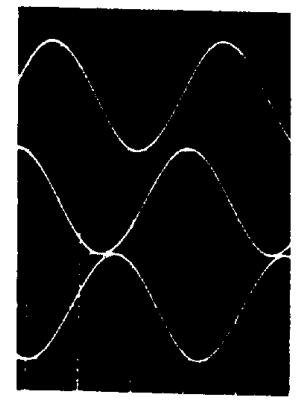

(b) $N=3$

Figure 4: (a) Numerical results for the case that $N=2,3$. ( $\left.\alpha=0.1, \beta=0.1, \gamma=0.1, \Delta \omega_{1}=0, \Delta \omega_{2}=0.001, \Delta \omega_{3}=0.002\right)$ (b) Experimental results for the case that $N=2,3$. ( $L=10 \mathrm{mH}$, $C=0.068 \mu \mathrm{F}, R=20 \Omega$, horizontal scale: $50 \mu \mathrm{sec} /$ div., vertical scale: $1.0 \mathrm{~V} /$ div. )

one negative resistor. In this system, according to the negative range of the coupling negative resistor, we can observe various interesting synchronization phenomena. Because this system tends to minimize the power consumption in the coupling negative resistor. Especially when $i-v$ characteristics of the coupling negative resistor are not third-power, but linear and negative, this system tends to be stable by producing the power in the coupling one. Because of the phase states is changed according to the negative range of the coupling negative resistor, we hope that our results can be applied to the design of some kinds of phase control systems.

\section{REFERENCES}

[1] H. Kimura and K. Mano, "Some Properties of Mutually Synchronized Oscillators Coupled by Resistances," Trans. IEICE, Vol. 48, No. 10, pp. 1647-1656 (in Japanese )

[2] T. Endo and S. Mori, "Mode Analysis of a Multimode Ladder Oscillator," IEEE Trans. Circuits Syst., Vol CAS-23, No. 2, pp. 100-113, Feb. 1976.

[3] T. Endo and S. Mori, "Mode Analysis of a TwoDimensional Low-Pass Multimode Oscillator," IEEE

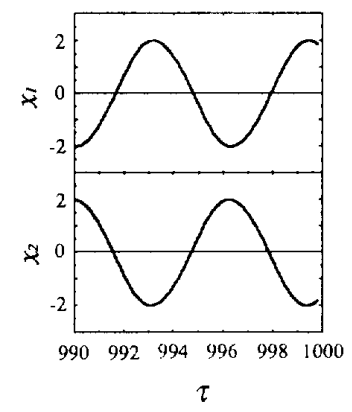

(a) $N=2$

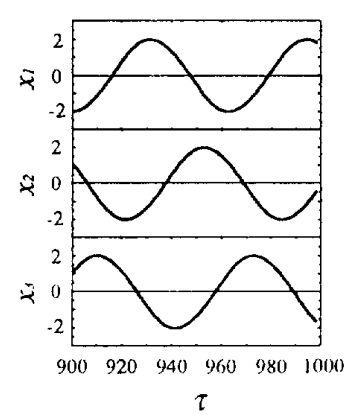

(a) $N=3$

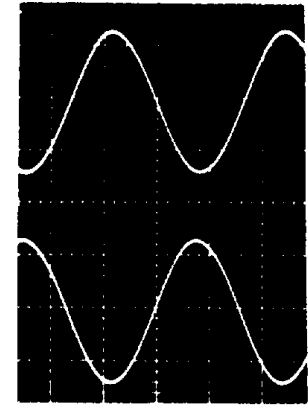

(b) $N=2$

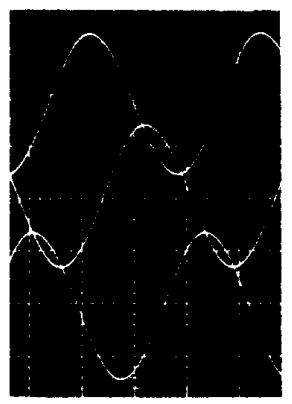

(b) $N=3$
Figure 5: (a) Numerical results for the case that $N=2,3$. ( $\alpha=0.1, \beta=0.1, \underline{\gamma=20}, \Delta \omega_{1}=0, \Delta \omega_{2}=0.001, \Delta \omega_{3}=0.002$ ) (b) Experimental results for the case that $N=2,3 .(L=10 \mathrm{mH}$, $C=0.068 \mu \mathrm{F}, R=20 \Omega$, horizontal scale: $50 \mu \mathrm{sec} /$ div., vertical scale: $10 \mathrm{~V} /$ div. )

Trans. Circuits Syst., Vol CAS-23, No. 9, pp. 517-530, Sep. 1976.

[4] T. Endo and S. Mori, "Mode Analysis of a Ring of a Large Number of Mutually Coupled van der Pol Oscillators," IEEE Trans. Circuits Syst., Vol CAS-25, No. 1, pp. 7-18, Jan. 1978.

[5] Y. Nishio and S. Mori, "Mutually Coupled Oscillators with an Extremely Large Number of Steady States," Proc. of ISCAS' 92, pp. 819-822, 1992.

[6] Y. Nishio and S. Mori, "On Coupled Oscillators Networks - for the Cellular Neural Network-,"Proc. of ISCAS' 93, pp. 2327-2330, 1993.

[7] S. Moro, Y. Nishio and S. Mori, "Coupled Oscillators with a Huge Number of Steady States - for a Structural Element of the Cellular Neural Network-.," Proc. of ECCTD' 93, pp. 27-32, 1993. 